\title{
ASPECTOS DE LA BIOLOGIA REPRODUCTIVA Y POTENCIAL PESQUERO DEL CAMARON DE RIO Macrobrachium amazonicum (Heller, 1862), EN LA RESERVA NACIONAL PACAYA-SAMIRIA, AMAZONIA PERUANA
}

\author{
Jorge AYARZA RENGIFO ${ }^{1,2}$; Rosa DEL AGUILA², Fernando ALCÁNTARA BOCANEGRA ${ }^{3}$; Carmen GARCÍA-DAVILA ${ }^{3}$ \\ 1 Dirección Regional de la Producción-DIREPRO-L, Iquitos Perú. E-mail jayarza@hotmail.com \\ 2 Becario de la Fundación Peruana para la Conservación de la Naturaleza, ProNaturaleza \\ 3 Instituto de Investigaciones de la Amazonía Peruana - IIAP. Programa para el Uso y Conservación del Agua y sus Recursos - \\ AQUAREC.E-mail: cdavila19@yahoo.com
}

\section{RESUMEN}

Fue estudiado la biología reproductiva y el potencial pesquero del camarón de río Macrobrachium amazonicum (Heller, 1862) en el sistema de lagos Macutari, Pungal y Grande de la Reserva Nacional Pacaya-Samiria (Amazonía peruana). El estudio fue realizado en base a 13,062 especimenes colectados entre mayo do 2003 a junio do 2004. Los resultados mostraron dimorfismo sexual en el tamaño del cefalotórax, las hembras son mayores que los machos (hembras $=30.9 \mathrm{~mm}$ y machos $=23.8 \mathrm{~mm}$ ). La proporción sexual hembra $:$ macho fue de 1.29:1. La especie presento reproducción continua, con picos reproductivos en los meses de noviembre y mayo, la fecundidad varió de 166 a 1,089 huevos por hembra, siendo que el numero de huevos presento poca relación con el tamaño de la hembra $(\mathrm{R}=0.46)$. La pesca del camarón en la reserva se realiza en forma artesanal mediante unas trampas denominadas "llica"; los meses de mayor actividad pesquera fueron noviembre y mayo, con una captura promedio por pescador de $31.34( \pm 17.5)$ camarones en un tiempo promedio de $58( \pm 25.58)$ minutos. La taza de conversión de camarón fresco a salado es de aproximadamente 18\%, la comercialización del camarón seco (denominado “camarón chino) es principalmente a la ciudad de Iquitos.

PALABRAS CLAVE: Camarón, Macrobrachium amazonicum, biología reproductiva, pesquería, Amazonía peruana.

\section{ASPECTS OF REPRODUCTIVE BIOLOGY AND FISHING POTENTIAL OF THE RIVER SHRIMP Macrobrachium amazonicum (Heller, 1862), IN THE NATIONAL RESERVE PACAYA-SAMIRIA, LORETO, PERU}

\begin{abstract}
We studied the reproductive biology and fishery potential of the river shrimp Macrobrachium amazonicum in the Macutari, Grande y Pungal lake systems, in the Pacaya-Samiria National Reserve (Peruvian Amazon). The study was based in 13,062 specimens collected between May 2003 and June 2004. The results showed sexual dimorphism in carapace size with females larger than males (females $=30.9 \mathrm{~mm}$ and males $=23.8 \mathrm{~mm}$ ). The male to female sex ratio was 1.29:1. Reproduction was continuous, with peaks in the months of November and May. Fecundity ranged from 166 to 1,089 eggs per female, and the number of eggs was not strongly related to female size $(\mathrm{R}=0.46)$. The traditional trap, or "Llica" is still used for Shrimp fishing in the National Reservation. Most fishing took place during the months of November and May, with an average catch per fisherman of $31.34( \pm 17.5)$ shrimp in an average time of $58( \pm 25.58)$ minutes. Dried, salted shrimp is converted to about about $18 \%$ the volume of fresh shrimp. The dried shrimp (called "Chinese shrimp) are primarily marketed within the city of Iquitos.
\end{abstract}

KEYWORDS: Shrimp, Macrobrachium amazonicum, reproductive biology, fishery, Peruvian Amazon 


\section{INTRODUCCIÓN}

La Amazonía es conocida como uno de los biomas con mayor diversidad biológica del mundo, posee un gran número de especies animales y vegetales y una amplia gama de ecosistemas. En contradicción con esto, la gente ribereña que habitan en contacto directo con estos ecosistemas, presentan poco desarrollo económico; pudiendo esto ser un claro indicativo de que hasta el momento no hemos encontrado el camino adecuado de potencializar el uso sostenido de nuestros recursos. Una de las posibles causas es el todavía limitado conocimiento de los mismos; los aspectos esenciales de su biología y ecología, de la forma de extracción, manejo sostenido y comercialización.

La Reserva Nacional Pacaya-Samiria (RNPS), es la segunda área protegida en extensión geográfica, y la más extensa en cuanto a área de bosque inundable protegido de la Amazonía peruana (INRENA, 2000). En esta extensa área la dirección de la RNPS, viene desde hace varios años atrás realizando esfuerzos para establecer y desarrollar proyectos de uso sostenido de especies emblemáticas de gran impacto y valor comercial como el paiche Arapaima gigas, la arahuana Osteoglossus biscirrosus y la tortuga Podocnemis unifilis. Sin embargo hasta el momento se presta aun poca atención a especies menos visibles como el camarón de río Macrobrachium amazonicum. Recurso hidrobiológico, que viene siendo utilizado en forma comercial en el sistema de lagos Macutari, Pungal y Grande por los pobladores ribereños del área de influencia de la reserva (tales como la comunidad de San Carlos, donde se llevó a cabo este estudio). En esta área este recurso es importante no solamente por su rol en la economía de la población, sino también por constituir una importante fuente de sustento alimentar. Hecho por el cual el conocimiento acerca de los rasgos de vida (reproducción, fecundidad, crecimiento etc.) e comercialización, son de vital importancia para a conservación y la sostenibilidad de este recurso.

En este sentido el presente estudio pretende contribuir al conocimiento del camarón de río Macrobrachium amazonicum en el área, cubriendo en gran parte vacíos de información sobre su biología reproductiva y potencial pesquero.

\section{MATERIAL Y METODOS}

Fueron realizadas colectas mensuales de especimenes del camarón de río Macrobrachium amazonicum (Figura 1) en el periodo comprendido entre mayo del 2003 a junio del 2004 en el sistema de lagos Macutari, Pungal y Grande en la Reserva Nacional PacayaSamiria (Figura 02), localizada aproximadamente a $450 \mathrm{~km}$ de la ciudad de Iquitos en la Amazonía peruana. Las colectas experimentales fueron realizadas con ayuda de una red de mano $(65 \mathrm{~mm}$ de diámetro) en un área de $400 \mathrm{~m}$ lineales. La abundancia fue determinada por el número de camarones colectados en 10 lances de la red de mano, analizando los resultados como Camarones/ Lance.

En el laboratorio, fue determinado el sexo de cada individuo con base en la presencia o ausencia del apéndice sexual masculino en el segundo par de pleópodos y se midió la longitud total del cefalotorax. La proporción de sexos fue determinada utilizando un test de chi - cuadrado $\left(\mathrm{X}^{2}\right)$ y las diferencias en la longitud total del cefalotorax de las hembras y los machos fueron comparados mediante un test "T". La variación en la longitud total del cefalotorax de los individuos durante el ciclo hidrológico, fue utilizada para estudiar la dinámica poblacional de esta especie en este sistema lagos.

El período reproductivo fue caracterizado por la presencia de hembras ovadas y recién desovadas (identificadas como tales por la presencia de residuos de huevos en los pleópodos y las pleuras alargada). La fecundidad se determinó en $10 \%$ de las hembras ovadas, en base al número total de huevos por hembra. Se analizó también la relación entre el número de huevos y la longitud total del caparazón por análisis de regresión simple. El volumen de huevos fue determinado según Medeiros et al. (1994), con la siguiente relación:

$$
\mathrm{V}=(\mathrm{A})^{2} \mathrm{X} 3,14 \mathrm{X} \mathrm{L} / 6
$$

Donde: $\mathrm{V}=$ volumen en $\mathrm{mm}^{3}, \mathrm{~A}=$ diámetro menor del elipsoide, $\mathrm{L}=$ diámetro mayor do elipsoide.

Para caracterizar la Pesquería, realizamos el acompañamiento a los pescadores que operan en la zona, durante un ciclo hidrológico, registrando la selección de las áreas y herramientas de pesca, Frecuencia de uso y eficiencia. Para determinar que la captura por unidad de esfuerzo (CPUE), se analizó el contenido de 100 trampas "Llica", estableciendo el número de camarones capturados, el tiempo de exposición y las profundidades en las que fueron colocadas las trampas. Fue acompañado también por el procesamiento de la captura, estableciendo las tazas de conversión de camarón fresco a seco y determinamos las rutas de comercialización.

\section{RESULTADOS Y DISCUSIÓN}

\section{PROPORCIÓN POR SEXOS Y DIMORFISMO SEXUAL EN EL TAMAÑO CEFALOTORAXICO}

De los 12120 individuos adultos colectados, 6820 
fueron hembras $(56.27 \%)$ y 5300 fueron machos $(43.73 \%)$, con una proporción sexual global hembra : macho de $1.3: 1$, este valor, no fue significativo en el test $\mathrm{X}^{2}$. La menor proporción fue observada en el mes de setiembre ( 0.8 hembra : 1 macho), en tanto que, las mayores proporciones de hembras con respecto a machos fueron observadas en los meses de junio y julio, con 2.4:1 y 1.8:1 respectivamente (Tabla 1). Gamba (1997) reporta para la especie una proporción sexual de 5.6:1 hembras con relación a machos en 292 individuos adultos (30 a $60 \mathrm{~mm}$ ) colectados en ecosistemas venezolanos. Esta diferencia encontrada en las proporciones de sexos de estos camarones podría estar reflejando diferentes estructuras poblacionales para esta especie o en su defecto diferencias en la colecta de campo entre los estudios. Es decir que los resultados podrían estar sesgados con la colecta de solamente adultos ya que esta especie como la mayoría de crustáceos presenta un carácter territorialista (clanes de machos con numerosas hembras), mientras que este comportamiento no es observado en las tallas menores, por lo cual una muestra poblacional con representantes de las diferentes marcas de clase asegura la obtención de una proporción sexual más real. Mayores proporciones de hembras con relación a machos ya fue observados también en otros crustáceos (Montreuil, et al., 1990; Odinetz-Collart, 1992; Odinetz-Collart, \& Enriconi, 1992; Medina \& Sobrino, 1994).

En cuanto a la longitud del cefalotórax, las hembras fueron de mayor tamaño que los machos, con una longitud total media de $30.9 \pm 0.3 \mathrm{~mm}$ y $23.8 \pm 0.1 \mathrm{~mm}$, respectivamente. La mayor longitud total observada fue de $54.9 \pm 0.5 \mathrm{~mm}$ para las hembras, en tanto que en los machos fue de $46.6 \pm 0.5 \mathrm{~mm}$. Esto podría estar asociado con el hecho de que las hembras se preparan físicamente para llevar los huevos y el cuidado de la descendencia, siendo que en los camarones las hembras tienen el abdomen, en promedio, más ancho y más largo que los machos (Rudolph, 1997; Ibarra \& Arana, 2012). Sin embargo algunos autores indican que el crecimiento en crustáceos es similar en ambos sexos hasta que alcanzan la madurez, a partir de la cual el crecimiento en las hembras puede ser menor debido a la producción e incubación de los huevos (MartínezMayen et al., 2000; Sánchez et al., 2008).

Los resultados muestran también que existe una estrecha correlación entre la longitud cefalotoráxica y el tamaño total, encontrándose un $\mathrm{r}=0.91$ para los machos, y de un $r=0.96$ para las Hembras (Figura 2).

\section{ABUNDANCIA}

De los 13062 camarones (hembras, machos y juveniles) analizados el $49.86 \%$ fueron colectados en la cocha Pungal, el $37.01 \%$ en la cocha Macutari y el $13.12 \%$ en la cocha Grande. La mayor abundancia (camarones/lance) fue registrada en el mes de agosto con 248.8 camarones/lance y la menor abundancia fue registrada en el mes de junio con 0.2 camarones/lance, en la cocha Pungal. Estas localidades presentaron la mayor abundancia de camarones en periodos de agua baja (Figura 3). Las mayores capturas se realizaron en los meses de agosto (2801) y octubre (2039), cuando el agua presentaba sus niveles más bajos. Estos resultados coinciden con los obtenidos en otros ecosistemas amazónicos como Venezuela (Gamba, 1997); Colombia, en la laguna Humacitas, río Meta (Medina \& Sobrino, 1994); y en la Amazonía central en los sistemas lacustres de la isla de Careiro, cerca de Manaus (Odinetz - Collart \& Moreira, 1993). La abundancia de este recurso en los meses de aguas bajas se podría atribuir a la retracción del medio físico, que trae como consecuencia una mayor concentración de individuos por falta de espacio para dispersarse.

\section{CARACTERIZACIÓN DE LA REPRODUCCIÓN}

\section{Período Reproductivo}

Del total de hembras adultas capturadas, el 25.83\% estuvo en estado ovígera. En las cochas Macutari, Pungal y Grande, se pudo observar hembras ovígeras de M. amazonicum durante todo el año, con algunos picos de mayor frecuencia en algunos meses del ciclo hidrológico (Figura 4). Reproducción continua durante todo el año fueron también reportados para esta especie en la Amazonía central (Brasil) por Odinetz - Collart (1988) y Odinetz - Collart \& Magalhães, (1994). En este estudio al igual que en la Amazonía central, el mayor numero de hembras ovígeras fue observado en el periodo en que las aguas comenzaron a incrementarse, es decir entre los meses de noviembre a diciembre del 2003 (49.24\%). Aunque en nuestro caso además observamos un pico reproductivo adicional durante las aguas altas de mayo del 2004 (16\%). La literatura reporta que el periodo reproductivo puede variar ligeramente de un año a otro en los camarones, debido principalmente a que son muchos los factores que pueden influir en este periodo, entre ellos, el nivel del río, la precipitación, la temperatura del agua, y otros factores medio ambientales (Medina \& sobrino, 1994; Walker \& Ferreira, 1985; Gamba, 1997; Odinetz-Collart, 1992; Odinetz-Collart, \& Enriconi, 1992).

\section{Fecundidad}

El tamaño medio de las hembras ovígeras fue de $43.3 \pm$ $0.3 \mathrm{~mm}$, la hembra ovígera de mayor tamaño presentó una longitud total de $50.9 \pm 0.3 \mathrm{~mm}$ y la de menor tamaño presentó $32.8 \pm 0.3 \mathrm{~mm}$ de longitud total. El 
promedio de huevos por hembra ovada fue de $535 \pm$ 13, la mayor fecundidad fue de 1089 huevos y la menor de 166. La relación estimada entre el número de huevos versus la longitud del cefalotórax de las hembras ovadas (Figura 5) fue muy débil en esta especie $(r=0.46, p=0.95)$. El número de huevos en los camarones, puede variar en función de la edad y del tamaño del individuo, así como por factores ambientales como la oferta alimenticia, la la temperatura y distancia geográfica con relación al mar (Odinetz - Collart \& Rabelo, 1996; Odinetz - Collart \& Magalhães, 1994; Magalhães \& Walker, 1988). En Macrobrachium amazonicum la fecundidad parece presentar una relación inversamente proporcional con la distancia geográfica del mar, así en el río Tocantins (afluente del Amazonas, cercano a Belén en Brasil, $120 \mathrm{~km}$ desde el mar) Odinetz-Collar \& Magalhães (1994) encontraron valores de fecundidad entre 258 a 5,706 huevos; en el lago del Rey, (aproximadamente $1225 \mathrm{~km}$ desde el mar) la fecundidad varió entre 365 a 2259 huevos, mientras que en este estudio la fecundidad fue de 166 a 1089 (aproximadamente 2940 $\mathrm{km}$ desde el mar). Esta aparente disminución en la fecundidad de una misma especie podría significar plasticidad para adaptarse a las pequeñas variaciones ambientales de su medio, con la finalidad de asegurar su suceso reproductivo. Así, en la baja Amazonía (Belem do Pará - Brasil) hay menor cantidad de sólidos en suspensión, las áreas de inundación y la riqueza en alimentos en las áreas cercanas a los estuarios son mayores (Odinetz - Collart \& Magalhães, 1994). En tanto que a medida que subimos la cuenca del río Amazonas la cantidad de sólidos en suspensión aumenta y las áreas de inundación y riqueza en alimentos disminuye.

Los huevos de Macrobrachium amazonicum presentan una forma elipsoidal, con un color verde oscuro al estado fresco y conservados presentan una coloración marrón lechosa. La longitud promedio fue $0.78 \mathrm{~mm} \pm 0.08$ (valor máximo 1.06 y mínimo $0.61 \mathrm{~mm}$ ) y ancho medio de $0.60 \mathrm{~mm} . \pm 0.05$ (valor máximo $0.73 \mathrm{~mm}$ y mínimo 0.49 ). El volumen medio fue de $0.15 \mathrm{~mm} 3 \pm 0.03$ (mayor volumen $0.29 \mathrm{~mm} 3 \mathrm{y}$ menor de $0.84 \mathrm{~mm} 3$ ). El volumen medio del huevo no presento correlación alguna con la longitud cefalotoráxica $(\mathrm{r}=0.091 ; \mathrm{p}=0.95)$. No en tanto esta demostrado que el volumen de los huevos de Macrobrachium amazonicum se incrementan con la distancia del sitio muestreado al océano, sugiriendo una progresiva divergencia de las poblaciones alejadas del mar con las poblaciones típicamente litorales (Odinetz - Collart \& Rabelo, 1996)

\section{CARACTERIZACIÓN DE LA ACTIVIDAD PESQUERA}

La pesca de camarones es considerada como actividad económica exclusiva en aproximadamente el 11.81\% de pescadores del área, siendo que el porcentaje restante de pescadores la consideran como una actividad parcial. Los lugares de captura son preferentemente debajo de los tamalones (islas flotantes), árboles sumergidos, vegetación arbustiva sumergida y macrófitas flotantes de los lagos. El arte de pesca empleado es una red conocida como "llica", cuya base inferior forma una canastilla rectangular de aproximadamente de $0.65 \mathrm{~m}$ de ancho por $1.20 \mathrm{~m}$ de largo, conformada por una red de plástico de $0.5 \mathrm{~mm}$ de abertura de malla, o en su defecto costal crudo; Esta red está sujeta a dos ramas de la liana Bombax munguba (punga), las cuales están cruzadas diagonalmente formando una asa entrelazada. Las "llicas" son camufladas con hojas de tal manera que simulan un colchón que proporciona sombra y refugio a los camarones contra sus depredadores. Las redes son colocadas a una distancia de uno a dos metros y a una profundidad aproximada de $0.5 \mathrm{~m}$ y dejadas allí por algunas horas.

En las 100 redes monitoreadas para el análisis de la captura por unidad esfuerzo se capturaron 9,683 camarones. La captura promedio fue de $31.34 \pm 17.5$ camarones por llica, con un tiempo promedio de 57.76 \pm 25.58 minutos. La extracción diaria en promedio de cada pescador en días fue de aproximadamente 3 a 5 kilos de camarón pilado por día, lo que representa una producción mensual aproximada de 45 a 75 kilos/ día por pescador. Considerando que esta actividad es desarrollada al menos tres veces al año en 15 pescadores, la proyección anual de la pesca de camarón sería de aproximadamente 1, 653 Kilos de camarón seco en un año. La estimación del número de camarones por kilo de camarón fresco reportó un promedio de $641.4 \pm 22$ camarones/kilo (camarones con tallas inferiores a $32 \mathrm{~mm}$; y pesos medios de 1.5 a 2 g) considerando este promedio, entonces podemos proyectar que la captura aproximada en los tres eventos al año seria de 5'890,190 camarones.

\section{PROCESAMIENTO，PRESERVACIÓN Y COMERCIALIZACIÓN}

Los camarones capturados son preservados por precocción por espacio de aproximadamente 20 a 25 minutos, en ollas con capacidad de $10 \mathrm{Kg}$, agregándose alrededor de $850 \mathrm{~g}$ de sal durante este proceso. Los camarones escurridos son extendiendo en el suelo sobre mantas de plástico que son expuestas al sol dos o tres días hasta que sequen y adquieran una apariencia "tostada', a continuación los camarones son colocados en costales en forma de cono donde son golpeados contra el suelo con lo cual se separa el exoesqueleto, luego el producto es tamizado para conseguir las "colitas" que son clasificadas según su 
tamaño y calidad. La tasa de conversión de camarón fresco a seco es muy baja, del $100 \%$ de camarones frescos $(10 \mathrm{Kg})$ al final del proceso de secado se obtiene solo un aproximado de $18 \%(1.8 \mathrm{~kg})$ de materia seca compuesto por las "colitas" para la comercialización. Este proceso también genera alrededor de $4.5 \mathrm{Kg}$. de "Polvillo" (exoesqueleto y restos de abdomen) que son desechados (aproximadamente $45 \%$ ).

En el proceso de comercialización cuenta con tres actores principales: a) los compradores rematistas primarios in situ que compran el producto dentro de las cochas, b) los compradores rematistas secundarios, que compran en las comunidades, y c) los comerciantes de los centros urbanos. Gran parte de la venta de camarón seco se realiza en la ciudad de Iquitos. En los últimos años una parte de la producción de camarones es comercializada en la zona de frontera con Brasil y Colombia y una pequeña es direccionada a su comercialización en la ciudad de Lima. En los centros urbanos los compradores rematistas secundarios comercializan el producto a diferentes precios, en Iquitos el precio del camarón seco fluctúa entre los S/. 30.00 soles x kilo (época de abundancia, setiembre a diciembre) a S/. 60.00 soles x kilo (época de escasez, febrero a abril). En la ciudad de Lima el precio aproximado es de $\mathrm{S} / .50 .00$ soles x kilo, en la ciudad de Manaus el precio es de \$. 25.00 dólares x kilo de camarón pilado. En los mercados de la región, se realiza la comercialización al menudeo, en bolsitas de polietileno, aproximadamente 10 gramos de camarón seco realizando la venta como 'Camarón chino" al precio de S/. 1.00 nuevo sol, con una ganancia neta .de S/. 70.00 nuevos soles por cada Kg. de camarón seco.

Tabla 1. Proporción mensual por sexos de ejemplares de $M$. amazonicum colectados en las tres cochas de estudio y prueba de Chi cuadrado para la determinación del nivel de significancia.

\begin{tabular}{|c|c|c|c|c|c|c|c|c|c|}
\hline \multirow{2}{*}{ MESES } & \multicolumn{2}{|c|}{$\mathrm{N}^{0}$ EJEMPLARES } & \multicolumn{2}{|c|}{$\%$} & \multicolumn{2}{|c|}{ PROPORCIÓN } & \multirow{2}{*}{$X^{2} C$} & \multirow{2}{*}{$\mathbf{X}^{2} \mathbf{T}$} & \multirow{2}{*}{ SIGNIFICANCIA } \\
\hline & 우 & $\sigma^{n}$ & Q & $\sigma^{n}$ & Q & $\sigma^{2}$ & & & \\
\hline Junio & 19 & 8 & 70.4 & 29.6 & 2.38 & 1 & 16.65 & 3.84 & - \\
\hline Julio & 35 & 19 & 60.8 & 39.2 & 1.84 & 1 & 4.67 & 3.84 & - \\
\hline Agosto & 1529 & 1207 & 55.9 & 44.1 & 1.27 & 1 & 1.39 & 3.84 & \\
\hline Setiembre & 242 & 301 & 44.6 & 55.4 & 0.8 & 1 & 1.17 & 3.84 & \\
\hline Octubre & 1161 & 768 & 60.2 & 39.8 & 1.51 & 1 & 4.16 & 3.84 & - \\
\hline Noviembre & 819 & 869 & 48.5 & 51.5 & 0.94 & 1 & 0.09 & 3.84 & \\
\hline Diciembre & 890 & 607 & 59.5 & 40.5 & 1.47 & 1 & 3.61 & 3.84 & \\
\hline Enero & 1125 & 697 & 61.7 & 38.4 & 1.61 & 1 & 5.38 & 3.84 & - \\
\hline Febrero & 404 & 364 & 52.6 & 47.4 & 1.11 & 1 & 0.27 & 3.84 & \\
\hline Marzo & 60 & 67 & 47.2 & 52.8 & 0.9 & 1 & 0.31 & 3.84 & \\
\hline Abril & 175 & 116 & 60.1 & 39.9 & 1.51 & 1 & 4.08 & 3.84 & - \\
\hline Mayo & 361 & 277 & 56.6 & 43.4 & 1.3 & 1 & 1.74 & 3.84 & \\
\hline TOTAL & 6820 & 5300 & 56.3 & 43.7 & 1.29 & 1 & 1.59 & 3.84 & \\
\hline
\end{tabular}

- = Valor significativo $(P \leq 0.05)$ 
FOLIA

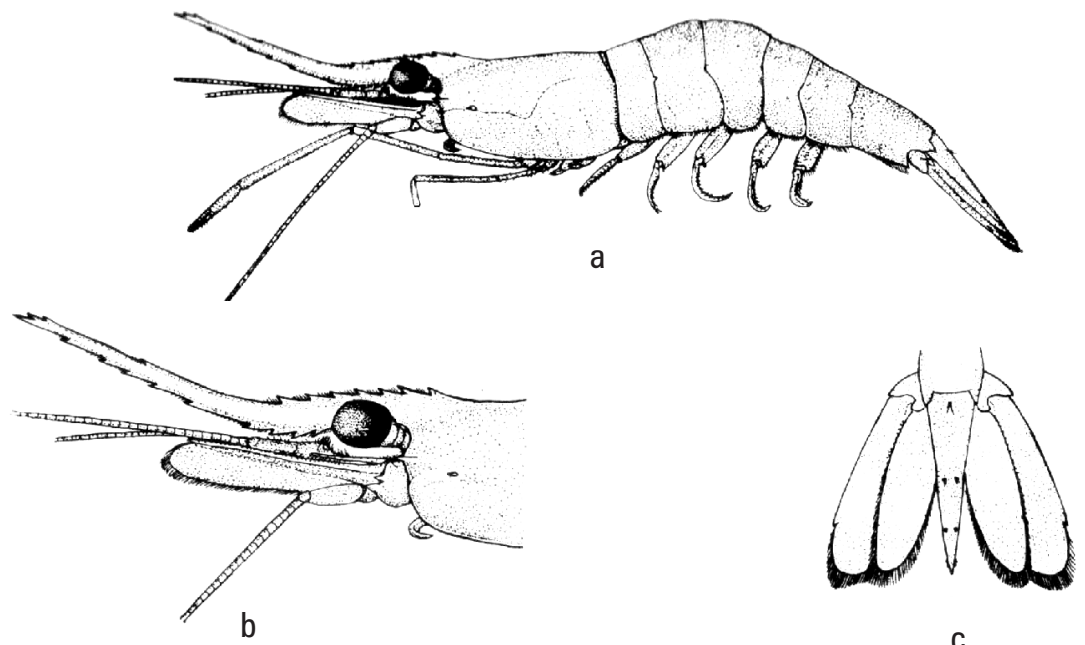

Figura 1. Ilustración del camarón de río Macrobrachium amazonicum: a) vista lateral; b) región frontal; c) telson y urópodos (Fuente: De Melo, 2003)

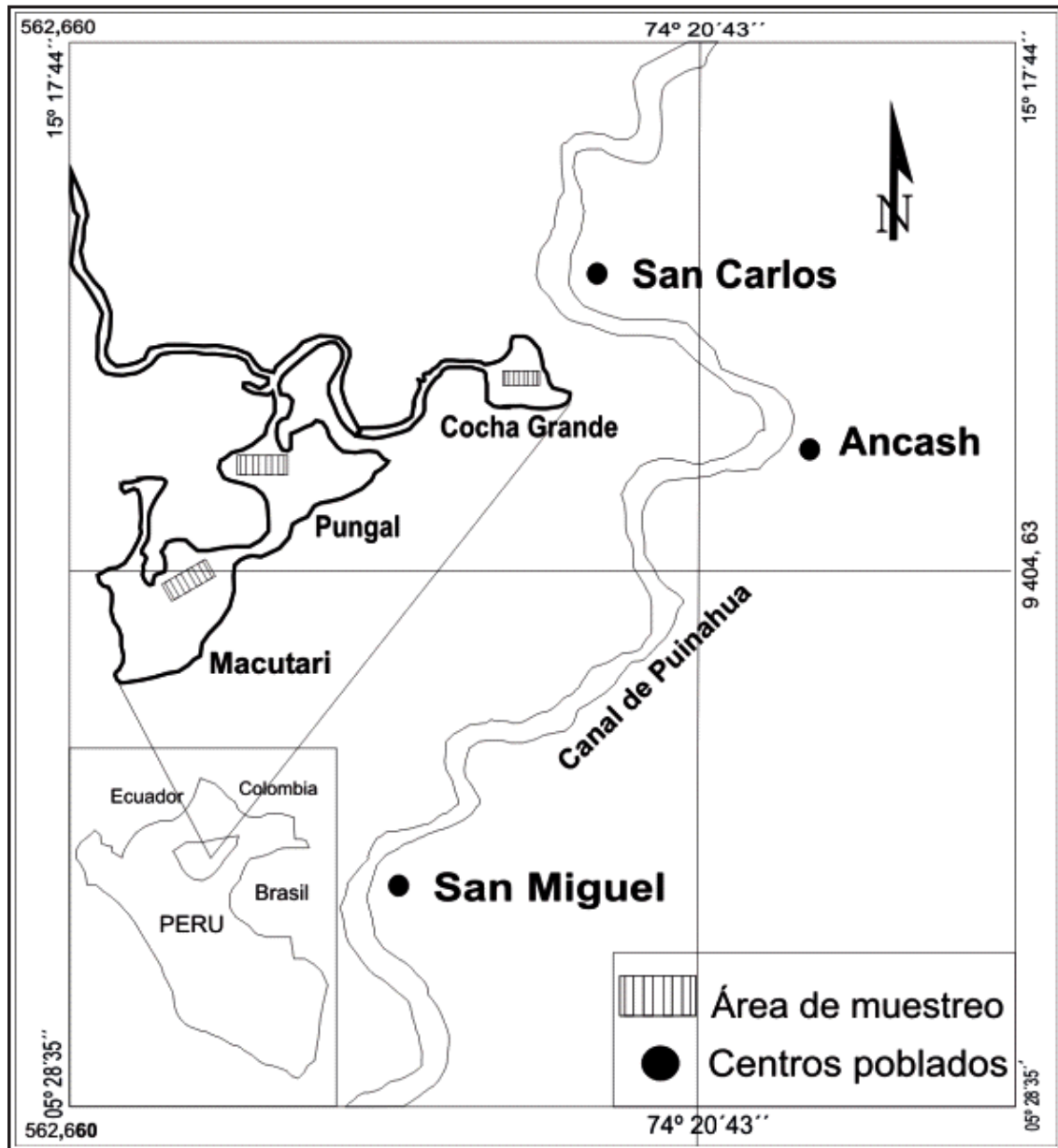

Figura 2. Área de estudio de Macrobrachium amazonicum en la Reserva Nacional Pacaya Samiria. Loreto, Perú. 


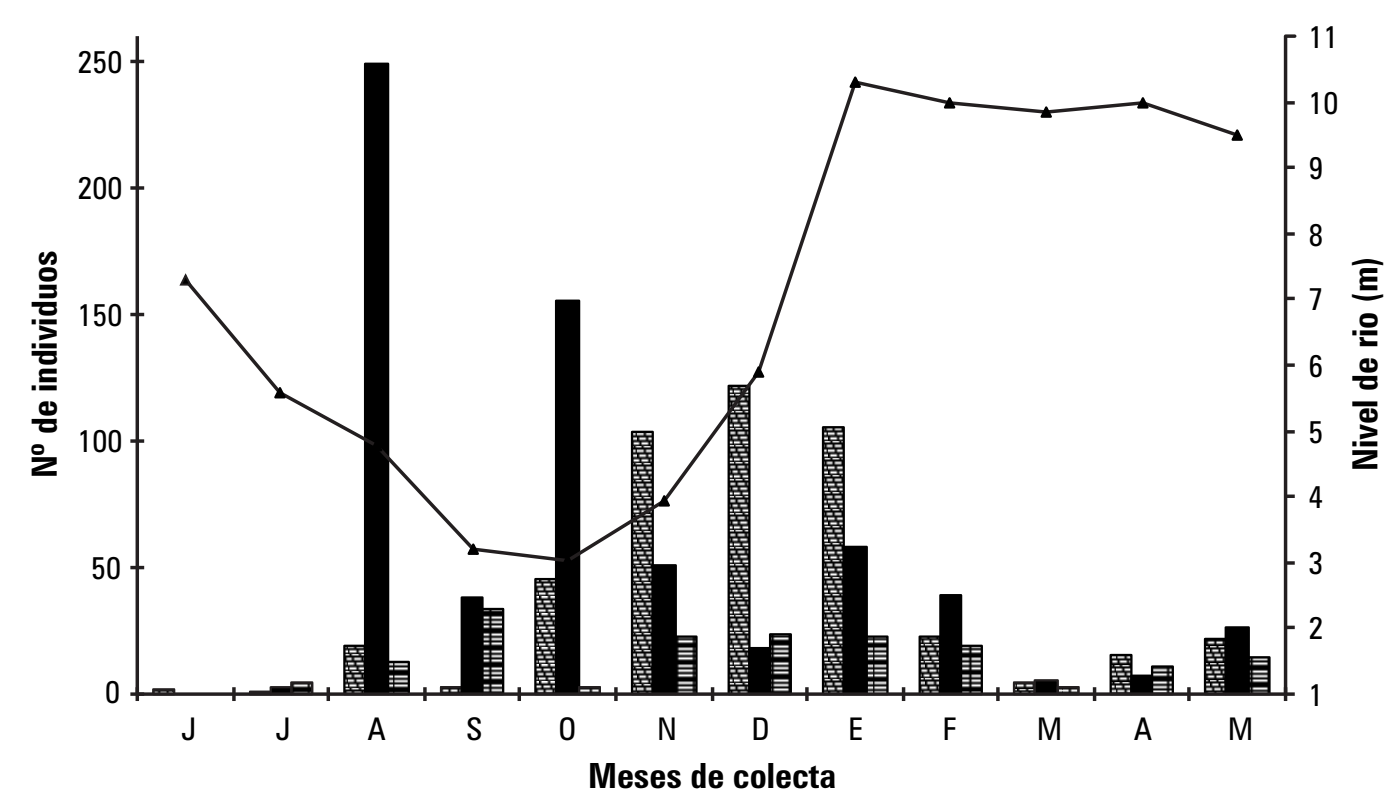

Macutari

Grande

— Nivel del río 2003 - 2004

Figura 3. Número de camarones colectados en las diferentes localidades de colecta durante un año.

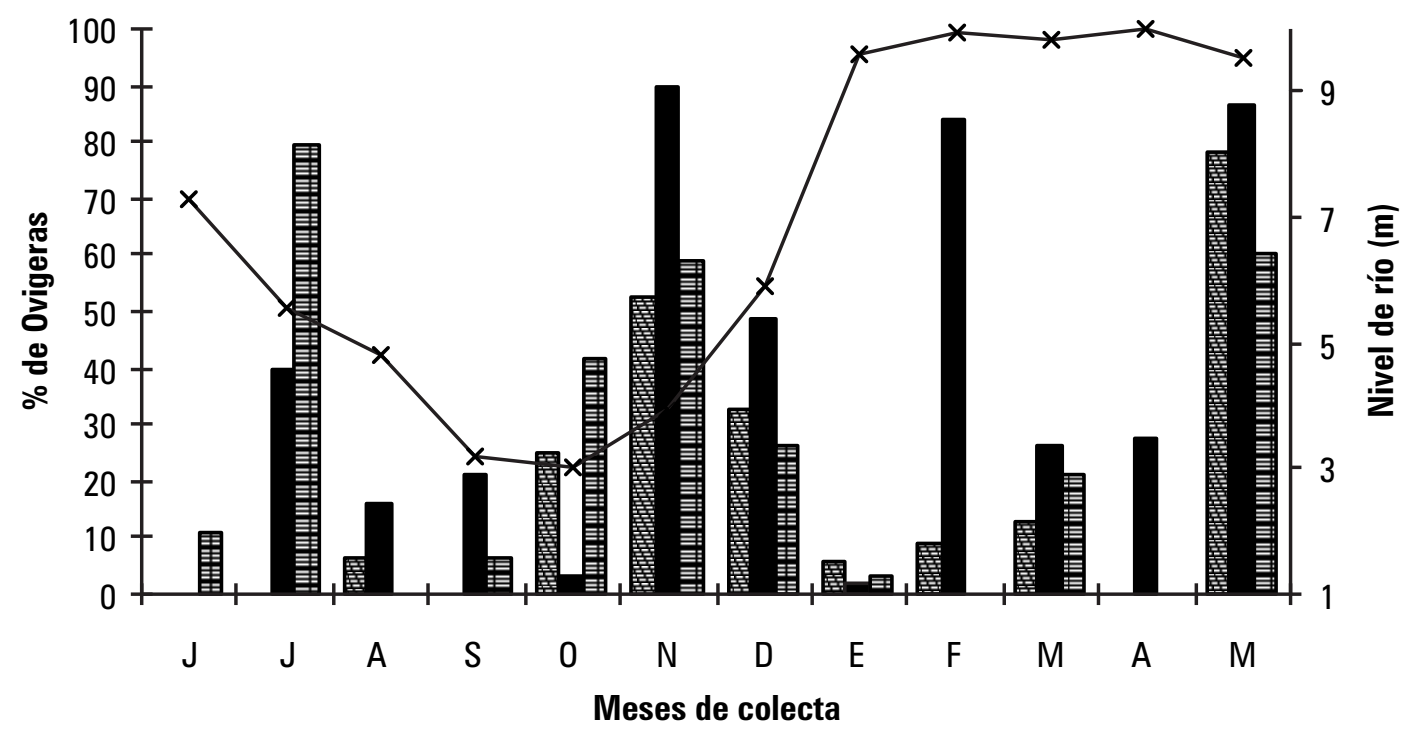

Pungal Gande $\quad x$ Nivel de río

Figura 4. Distribución mensual de hembras ovígeras de Macrobrachium amazonicum y su relación con el nivel del río, en los meses de colecta. 


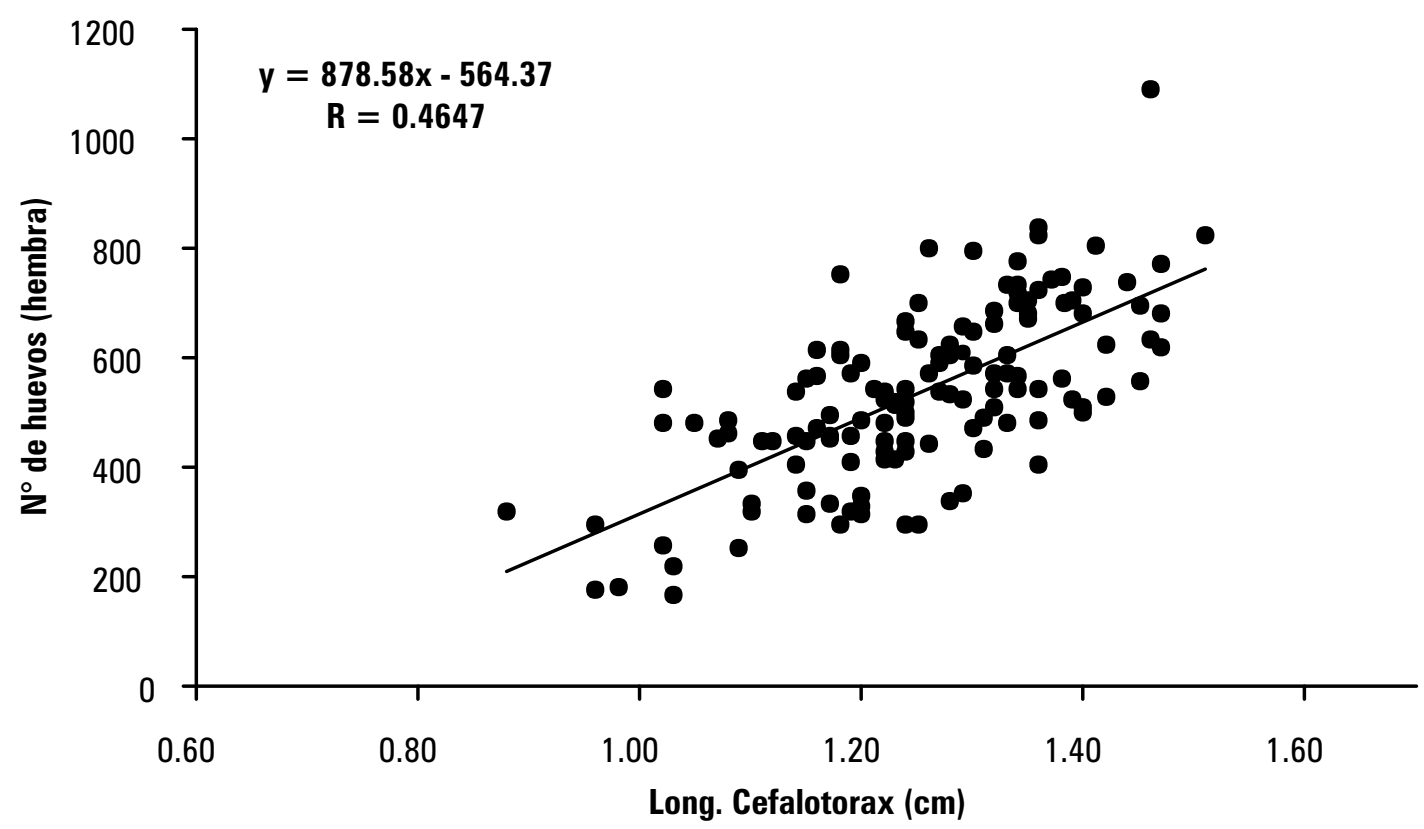

Figura 5. Relación longitud del cefalotórax versus el número de huevos en las hembras ovadas de Macrobrachium amazonicum estudiadas.

\section{AGRADECIMIENTOS}

Los autores agradecen a la Fundación Peruana para la Conservación de la Naturaleza, ProNaturaleza, que a través del Proyecto Mac Arthur, nos concedió el financiamiento para la realización de este estudio.

\section{BIBLIOGRAFÍA CITADA}

Gamba, L. 1997. Biología Reproductiva de Macrobrachium jelski (Miers, 1877) y Macrobrachium amazonicum (Heller, 1862) en Venezuela (Crustácea, Decapoda, Palaemonidae). Acta Científica Venezolana. 48: 19-26.

Ibarra, M.; Arana P. 2012. Biological parameters of the burrowing crayfish, Parastacus pugnax (Poeppig, 1835), in Tiuquilemu, Bío-Bío Region, Chile. Latin american journal of aquatic research, 40(2): 418-427.

Instituto Nacional de Recursos Naturales (INRENA). 2000. Plan Maestro de la Reserva Nacional Pacaya Samiria. Loreto-Perú. 153 pp.

Martínez-Mayen, M.; Román-Contreras, R. 2000. Aspects of the reproduction of Atya margaritacea A. Milne-Edwards, 1864 (Decapoda, Atyidae) in a population from the Mexican Pacific. Crustaceana 73:913-923.
Medina, R ; Sobrino, A. 1994. Contribución a la Ecología y Cultivo de larvas del camarón de agua Dulce Macrobrachium amazonicum (Heller, 1862) (Decapoda: Palaemonidae). Tesis para optar el Titulo de Biólogo. Universidad Nacional de Colombia.

Medeiros, N.; Magalhães, C ; Odinetz Collart, O. 1994. Caracterização morfologica e reprodutiva do camarão Macrobrachium inpa (Decapoda, Palaemonidae). In: anais- $46^{a}$ Reunião Anual da SBPC, Universidade Federal do Espírito Santo. p. 826-836.

Montreuil, V. H.; Maco, J. ; Tello, S.; Ismiño, R. ; Sánchez, H. 1990. Cuadro medio ambiental de la cocha Carocurahuaite y las posibilidades de explotación del camarón Macrobrachium amazonicum. Folia Amazónica, 2:99-121.

Magalhães, C. ; Walker, I. 1988. Larval development and ecological distribution of central Amazonian Palaemonidae shrimps (Decapoda: Caridea). Crustaceana. 55 (3) 279-292.

Odinetz-Collart, O. 1988. Aspectos ecológicos do Camarão Macrobrachium amazonicum (Heller, 1962) no baixo tocantins (PA - Brasil) Memorias de la Sociedad de ciencias Naturales La Salle, XLVIII, , suplemento: $341-353$. 
Odinetz-Collart, O. 1992. Ecologia e potencial pesqueiro do camarão-canela Macrobachium amazonicum na bacia amazónica In: Bases Cientificas para Estratégias de Preservação e Desenvolvimento da Amazônia. Instituto Nacional de Pesquisas da Amazônia (INPA). 2: 147- 166.

Odinetz-Collart, O. \& Enriconi, A. 1992. Ecologia do camarão Macrobrachium nattereri (Heller, 1862) nos Igarapés do rio Negro, Amazônia central. IV Congresso Brasileiro de Limnologia, Manaus, Brasil 07 al 11 de Setembro de 1992. Abstracts.

Odinetz-Collart, O. ; Moreira, L. 1993. Potencial pesqueiro de Macrobrachium amazonicum (Ilha do Careiro): Variacão da abundância e do comprimento. AMAZONIANA XII(3/4):399 - 413.

Odinetz-Collart, O; Magalhães, S. 1994. Ecological constraints and life history strategies of Palaemonidae pawns in Amazonian. Verhandlungen der Internationale Vereinigung Limnologie. 25:2460-2467.
Odinetz-Collart, O; Rabelo, H. 1996. Variation in egg size of the fresh-water prawn Macrobrachium amazonicum (Decapoda. Palaemonidae). Jounal of Crustacean Biology 16(4):684-688.

Rudolph, E. 1997. Intersexualidad en el camarón excavador Parastacus pugnax (Poeppig, 1835) (Decapoda; Parastacidae). Invest. Mar., 25: 7-18.

Sánchez, j.; Alvarez, R.; Ramírez, J. 2008. Crecimiento y reproducción del camarón Atya margaritacea (Decapoda: Atyidae) en el Río Presidio, Sinaloa, México. Revista de Biología Tropical, 56 (2): 513-522.

Walker, I.; Ferreira, M. J. N. 1985. On the population dynamics and ecology of the shrimp species (Crustacea, Decapoda, Natantia) in the central Amazonian river Taruma-Mirin. Eoecología, 66:264-270. 